\title{
Effect of individual counseling on pain quality in the women with cyclic mastalgia: a clinical trial
}

\author{
S. Fakhravar ${ }^{1}$, N. Bahrami ${ }^{1}, \underline{\text { F. Olfati }}^{2}$
}

\begin{abstract}
${ }^{1}$ School of Nursing and Midwifery, Qazvin University of Medical Sciences, Qazvin, Iran
${ }^{2}$ School of Nursing and Midwifery, Metabolic Diseases Research Center, Qazvin University of Medical Sciences, Qazvin, Iran

Corresponding Address: Forozan Olfati, Schools of Nursing and Midwifery, Qazvin University of Medical Sciences, Qazvin, Iran

Tel: +98-912-1826082; Email: papoy6olfati@yahoo.com

Received: 3 Apr 2018; Accepted: 30 Jun 2018
\end{abstract}

\section{Abstract}

Background: Despite the high prevalence of cyclic mastalgia and disagreement about its therapeutic methods, there is a lot of ambiguity about breast pain yet.

Objective: This study aimed to investigate the effect of individual counseling on the quality of pain in the women with cyclic mastalgia.

Methods: This randomized-controlled trial study was conducted in 2017 on eighty eligible women with cyclic mastalgia that had referred to Health Centers in Karaj, Iran. The subjects were randomly assigned to two groups; intervention and control. Four individual counseling sessions were held for intervention group. With a special visual analog scale for pain and Cardiff's breast pain chart, cyclic mastalgia was diagnosed. Pain was assessed before and after consultation with McGill pain quality questionnaire. T-test and ANCOVA were used to examine the means of pain quality before and after the intervention.

Findings: Demographic results including, marital status, educational level, occupation, spouse's education and the husband's job were not significant. Also, the history of lactation, surgery, breast sampling, benign breast disease, nipple discharge and breast injury were no significant between two groups as the chi-square test. But, after the intervention, the McGill mean score test in all of pain dimensions showed a significant difference between two groups $(\mathrm{P}=0.001)$.

Conclusion: This study showed that counseling can lead improvement of pain quality indices in affecting women. As the result, counseling can be suggested as a suitable treatment for mild to moderate pain.

Keywords: Mastalgia, Counseling, Pain, Breast

Citation: Fakhravar S, Bahrami N, Olfati F. Effect of individual counseling on pain quality in the women with cyclic mastalgia: a clinical trial. J Qazvin Univ Med Sci 2018; 22(4): 22-30. 


\title{
تأثير مشاوره فردى بر كيفيت درد زنان مبتلا به ماستالزى دورهاى: يك كار آزمايى بالينى
}

\author{
سولماز فخرآور '، دكتر نسيم بهرامى'، دكتر فروزان الفتى'
}

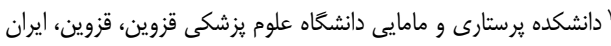

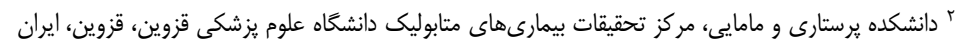

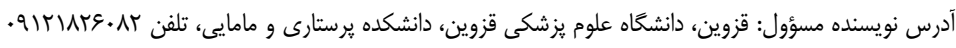

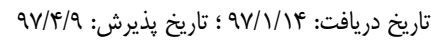

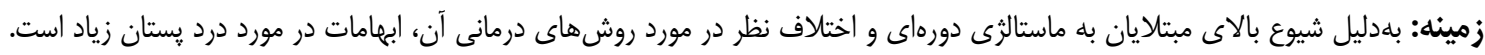

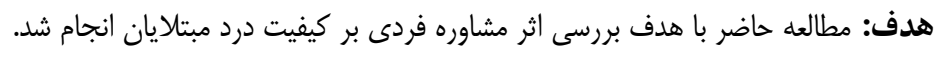

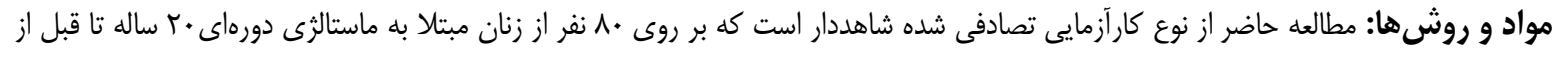

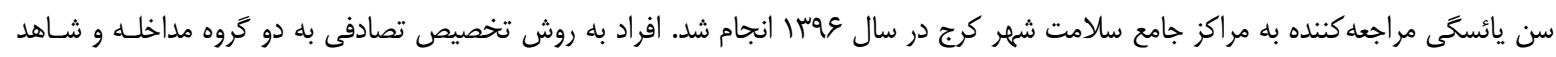

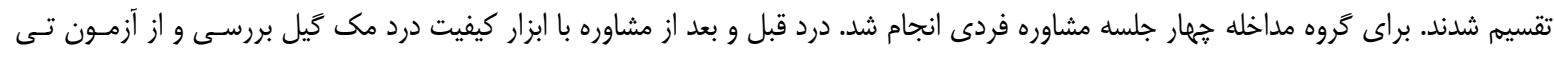

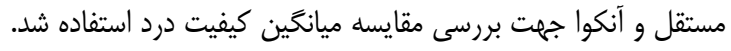

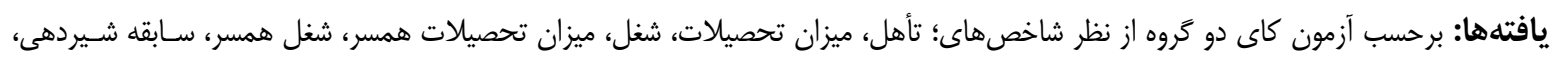

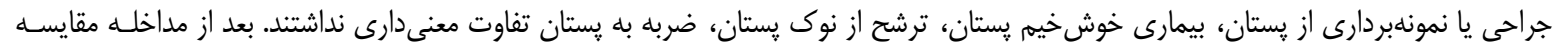

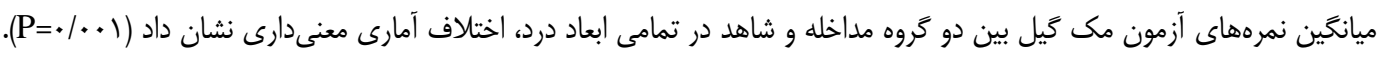

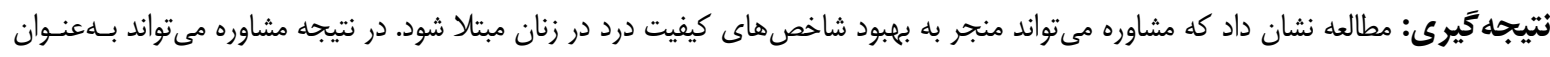
يك روش درمانى مناسب براى دردهاى خفيف تا متوسط ييشنهاد شوداد

كليدوازهها: ماستالزى، مشاوره، درد، پِّتان

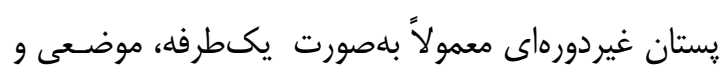

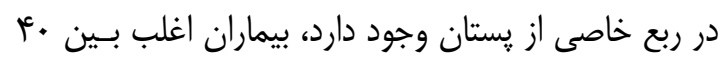

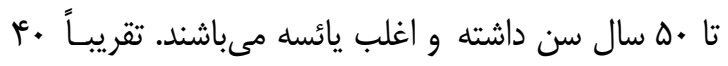

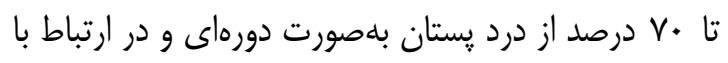

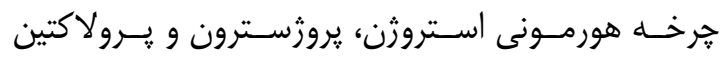
مىباشد.) علت درد يستان هنوز ناشناخته است، دلايلـى مانـــــ

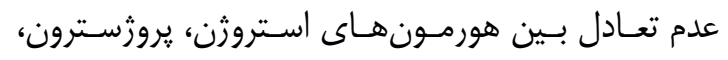

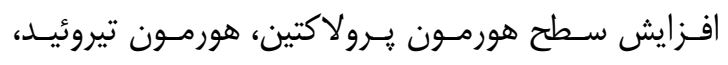

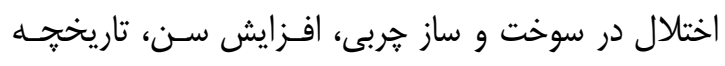

\section{楚}

درد پِتان يكى از شايعترين دلايل نكَرانى زنان است

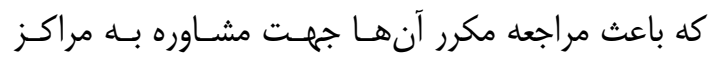

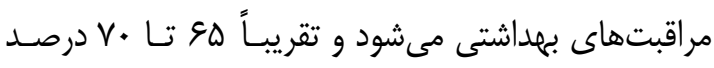
زنان واقع در سنين بارورى در طول عمر خود به آنه آن مبتلا

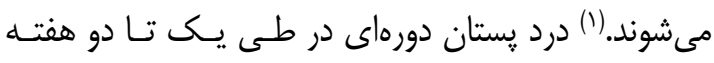

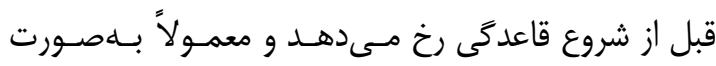

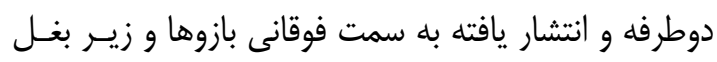

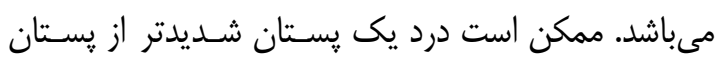

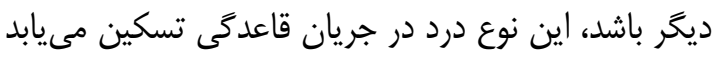

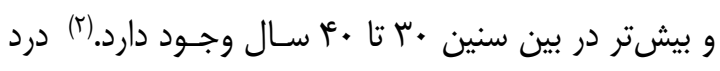


يروستاكلاندينهاسـت.(I) درمـانهـاى غيردارويسى نظيـر

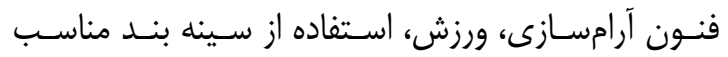

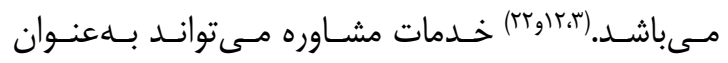

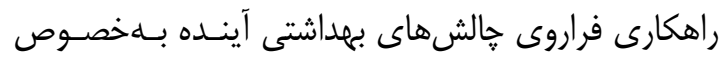

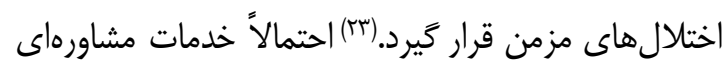
با مكانيسم كاهش ترس، اضـطراب، نغخرانسى و بـا ايجـاد اعتماد به نفس و ارتقاى درى از بيمارى مى توانسـ آسـتانه

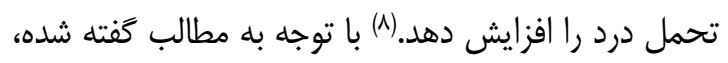

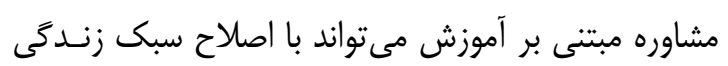

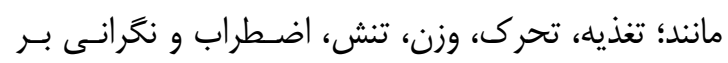
كيفيت درد مؤثر باشد.

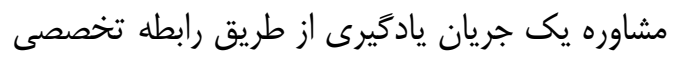

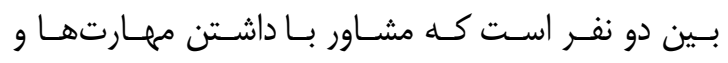

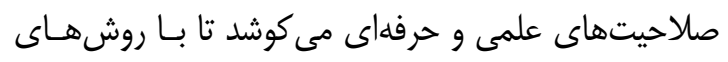

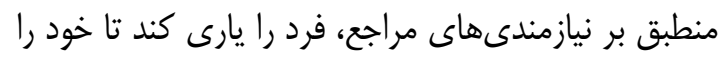

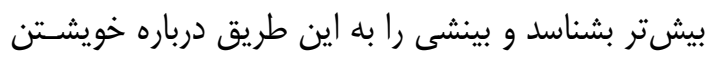

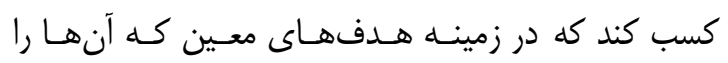

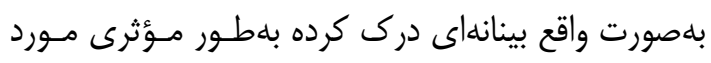

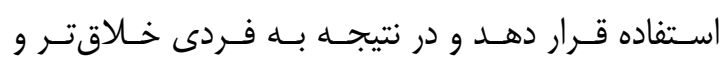
شادمانتر در جامعه خود تبديل شود..(TF)

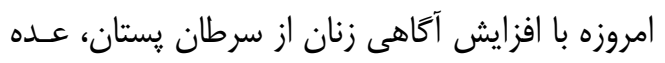

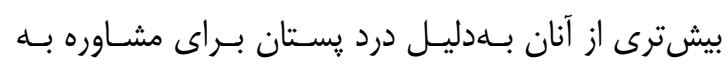

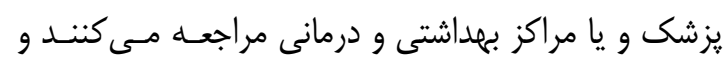

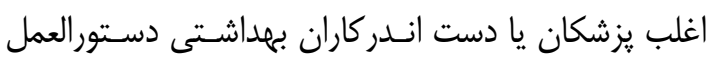

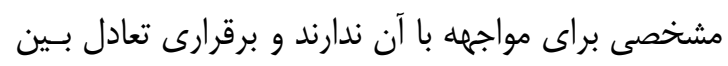

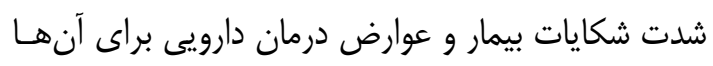

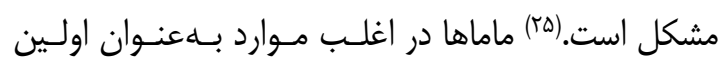

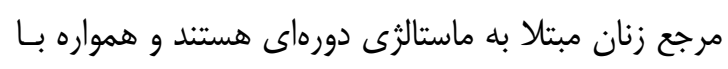

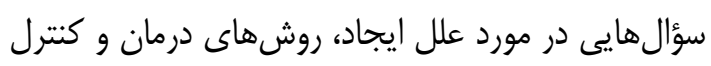

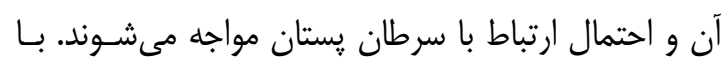

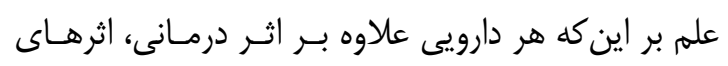

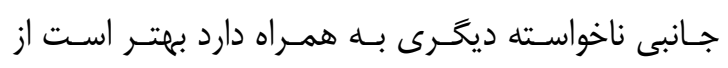

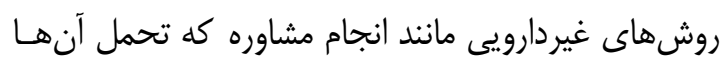

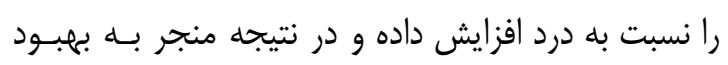

سندرم ييش از قاعدَّى، تنش، اضطراب، افسـردگى، سـوء

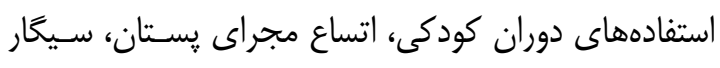
كشيدن و مصرف كافئين مطرح است. (ז-1-أ)

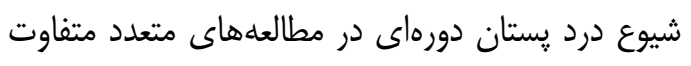

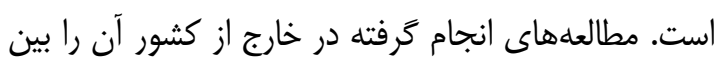

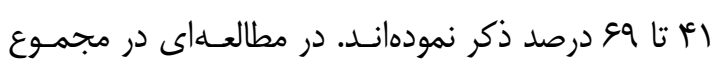

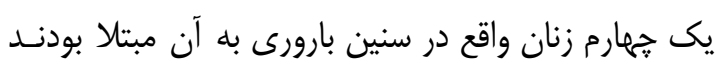

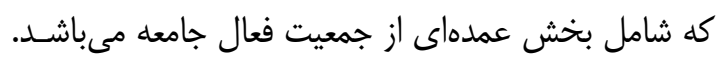

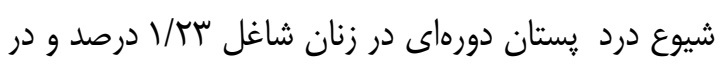

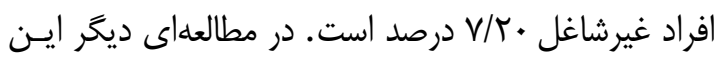

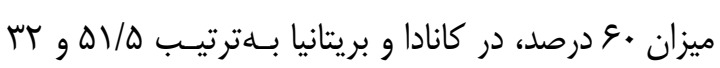

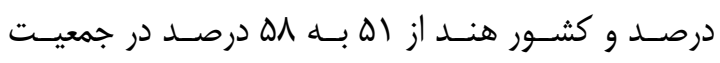

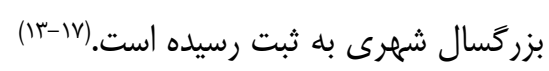

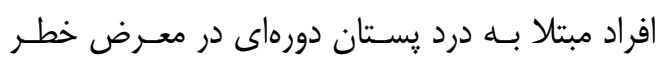

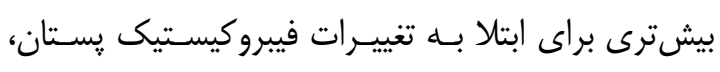

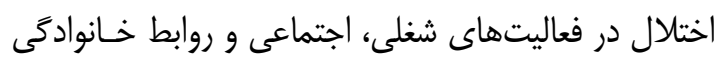

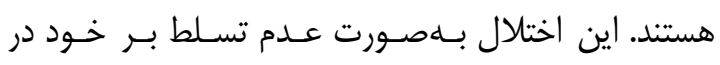

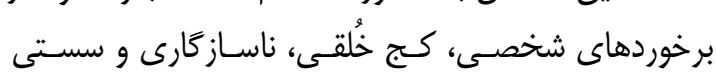

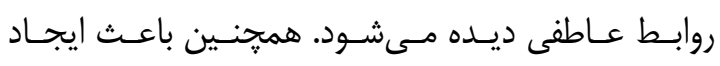

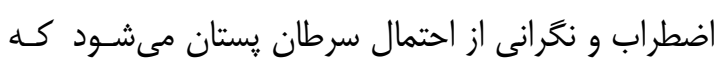

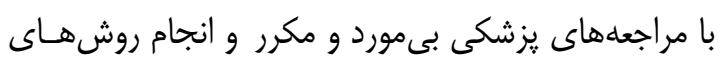

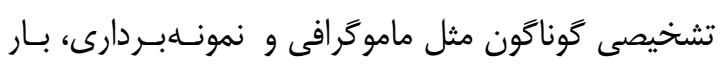

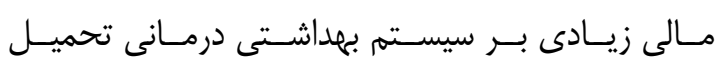

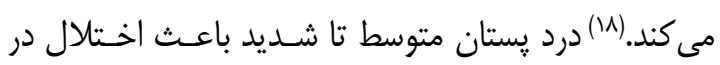

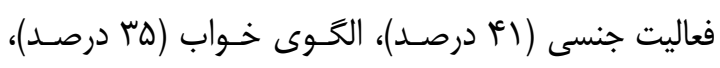

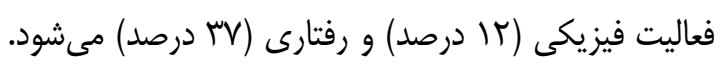

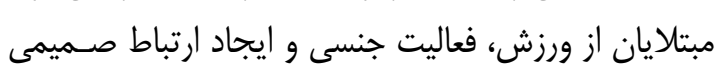

اجتناب مى كنند كه بر كيفيت زندگى مؤثر است. (آ-19)

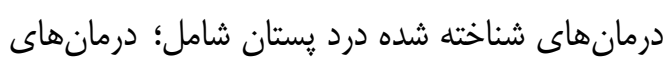

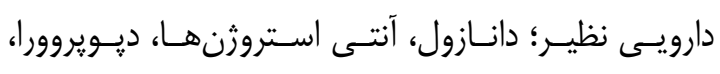

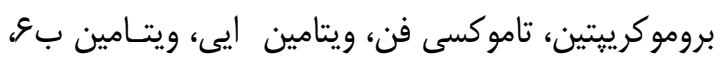

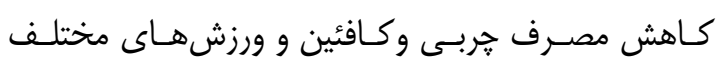

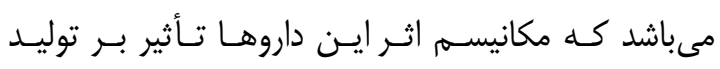

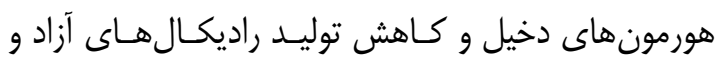


روش تصادفى از اين مراكز انتخاب شدند. جهت اختصاص

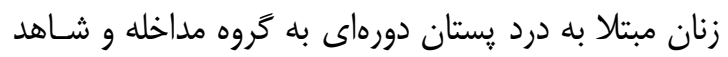

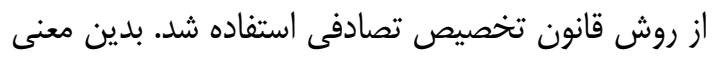

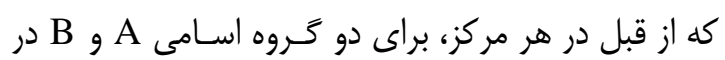

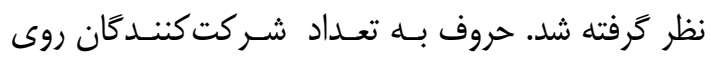

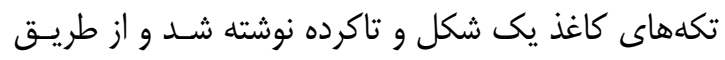

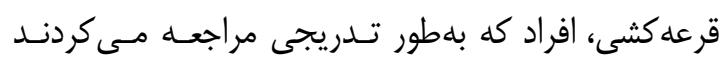

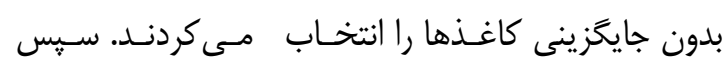

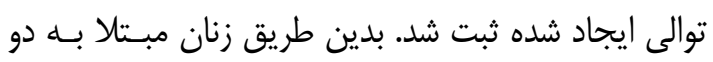
كروه مداخله و شاهد تقسيم شدند.

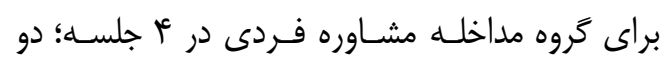

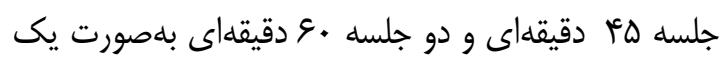

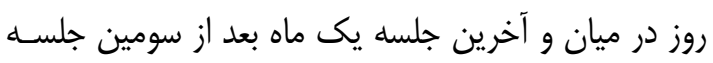

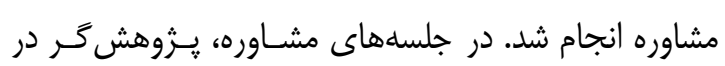

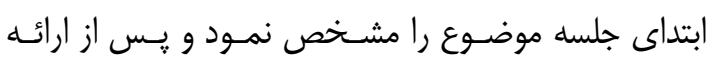
اطلاعات در مورد موضوع به زبانى ساده از طريـق سـؤال

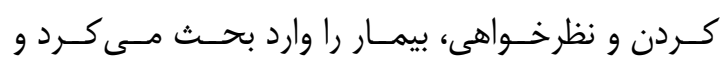

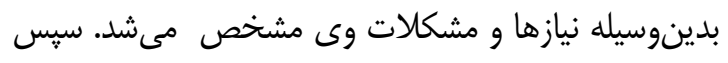

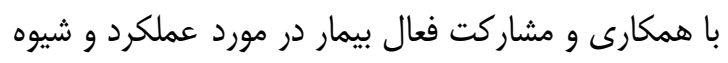

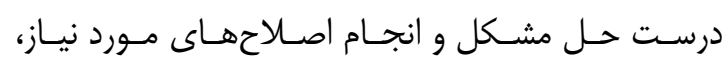

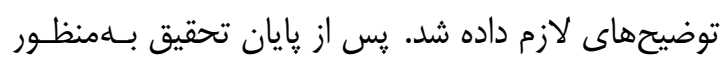

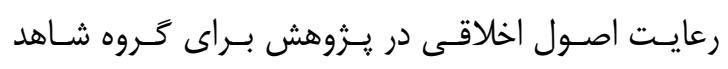

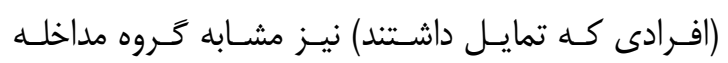
جلسههاى مشاوره فردى بركزار شد.

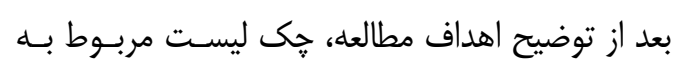

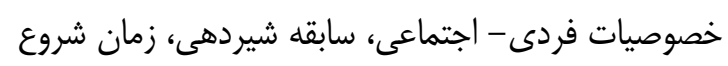

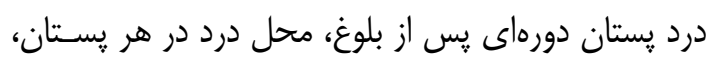

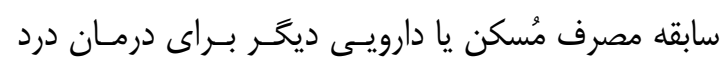

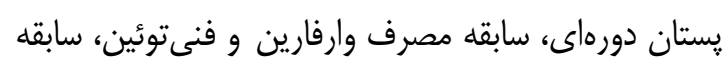

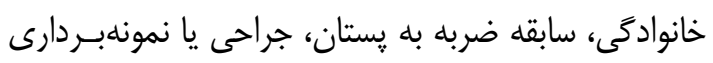

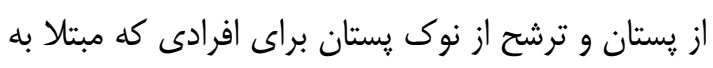

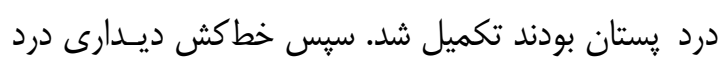

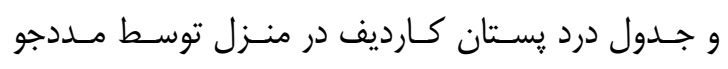

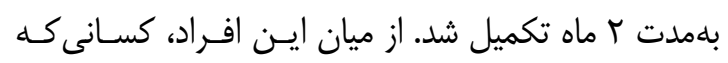

كيفيت زندكَى آنها مسىشـود، اسـتفاده حَردد. بنـابراين، مطالعه حاضر با هدف بررسى تأثير مشاوره فردى بر كيفيتيت زندگى زنان مبتلا به ماستالزى دورهاى انجام شد.

\section{مواد و ورشها:}

مطالعه حاضر از نوع كارآزمايى تصادفى شده شاهددار

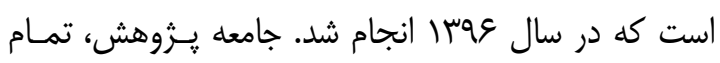

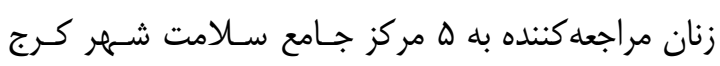

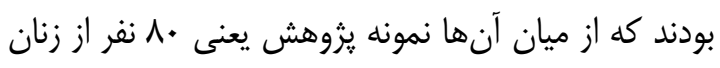

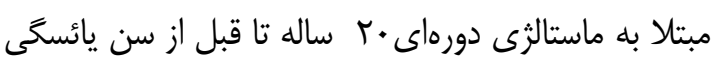

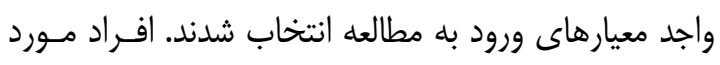

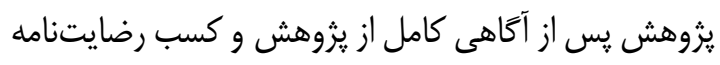
كتبى و تمايل به شركت، وارد مطالعه شدند. شر ايط ورود به مطالعه؛ زنـان داراى معيـار درد سـينه

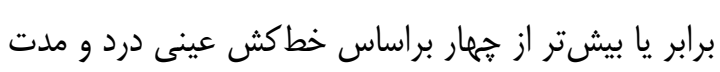

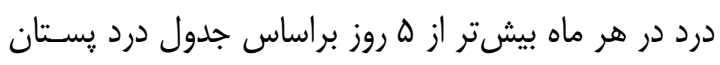

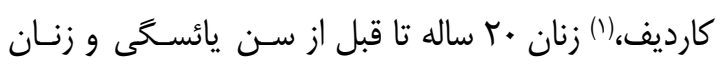

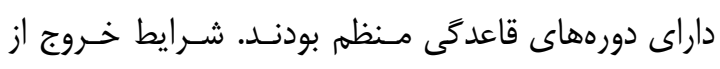

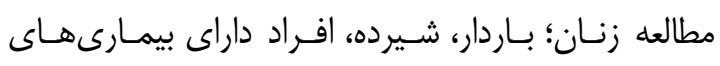

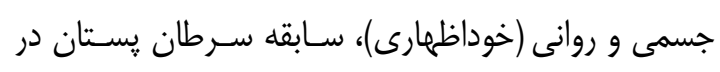

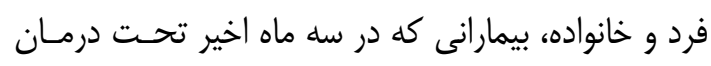

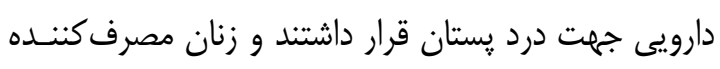

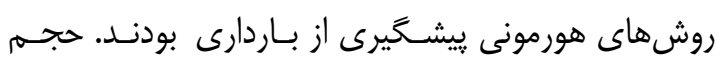

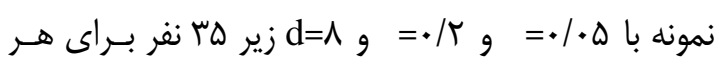

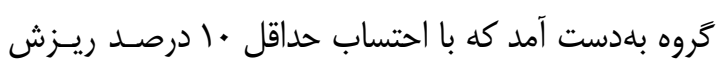

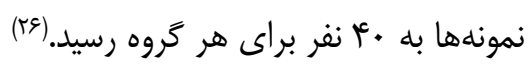

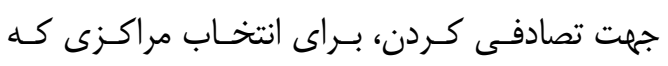

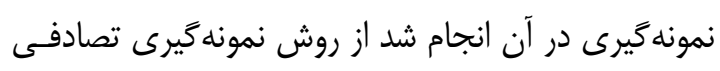

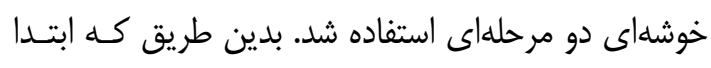
ليستى از مراكز جامع سلامت دانشكاه علوم يزشكى البـرز

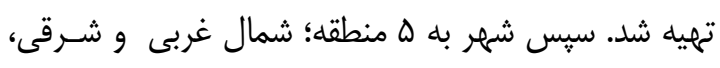

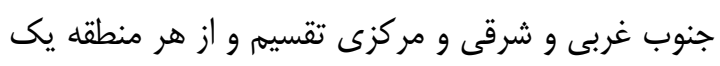

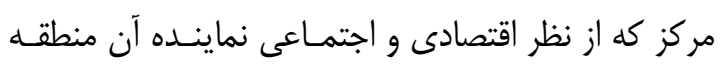

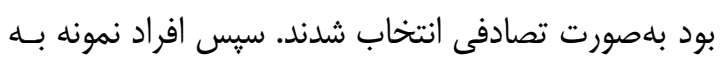


(نمره f تا V نشانه درد متوسط و V تا 9 نشانه درد شـديد

ץ- جَى ليست اطلاعات فردى -اجتماعى سـؤال هـايى در مورد خصوصيات جمعيتشناختى، سابقه شـيردهى، ضـربه

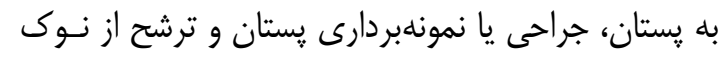

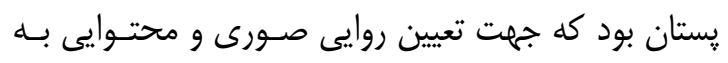
نظر ه نفر از اعضاى متخصص گروه مامايى رسيد و مورد تأييد قرار كرفت.

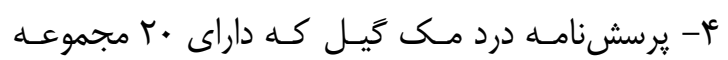
عبارت است و هدف آن سنجش درك افراد از درد در ابعاد

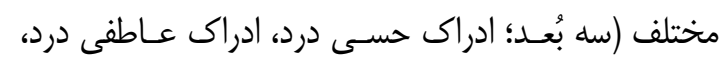

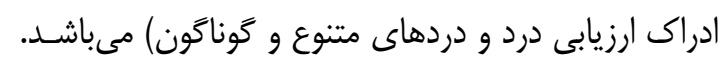

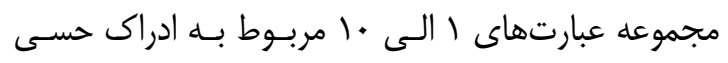

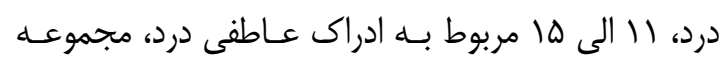

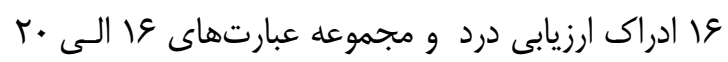

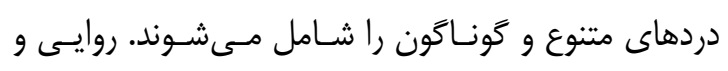

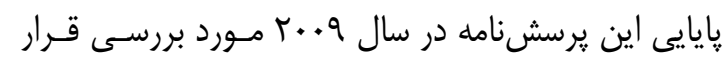

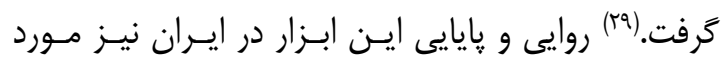

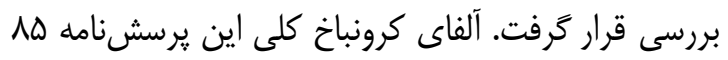

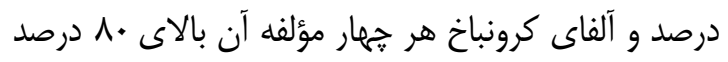

$$
\text { كزارش شد.) }
$$

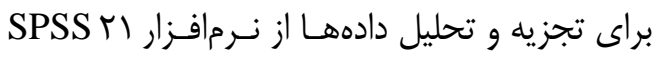

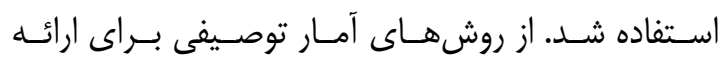
اطلاعات كلى، جهت مقايسه بين دو گروه مداخله و شاهد

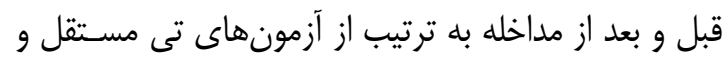

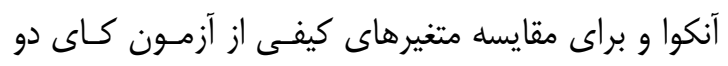
استفاده شد. سطح معنى دارى كمتر از هـ • • تعيين گرديد.

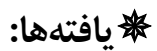

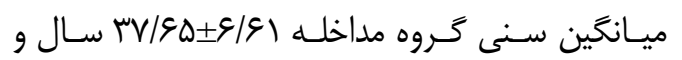

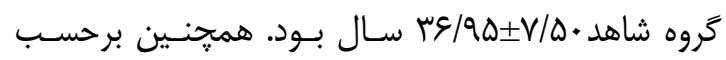

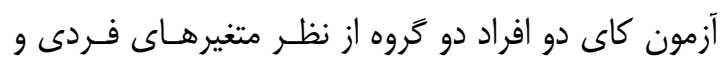

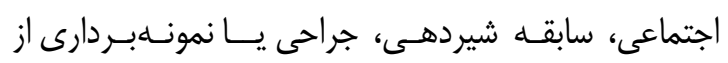

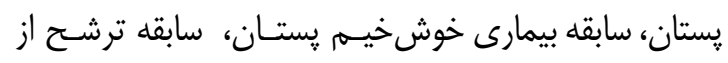

شدت درد مساوى و يا بيش از أو طول مــدت درد آنهـا بيش از ه روز بود وارد مطالعه شدند. قبل از انجام مداخلـه مانه

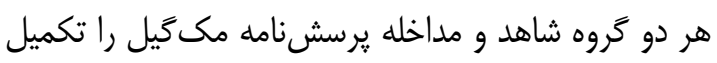

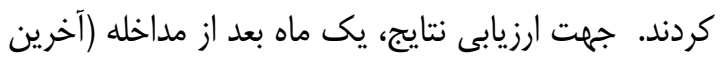

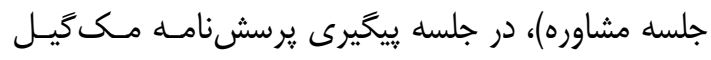
توسط دو گروه تكميل شد.

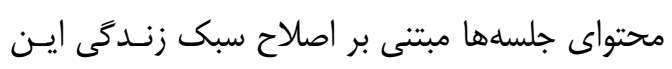

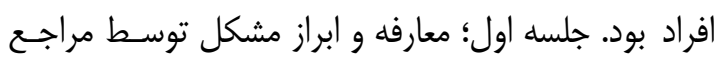

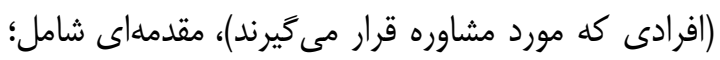

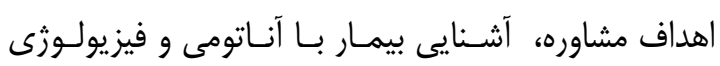

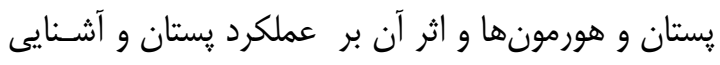

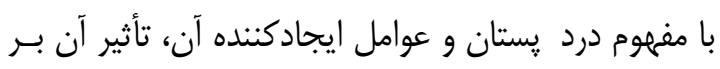

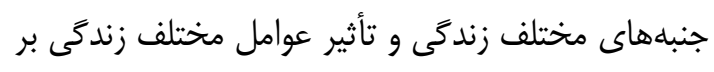

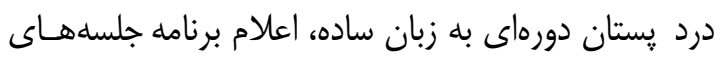

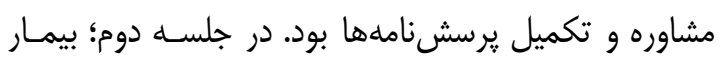

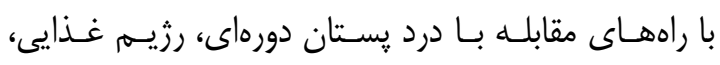

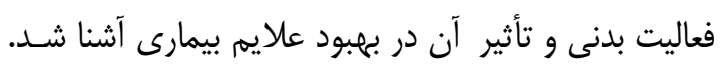

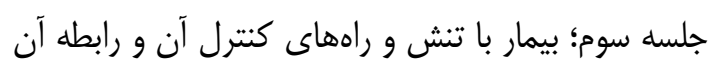

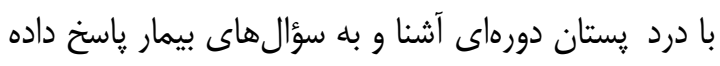

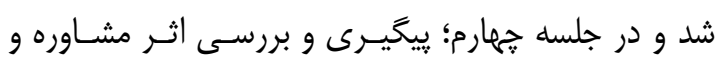

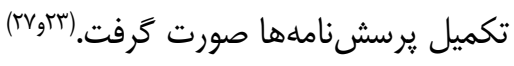

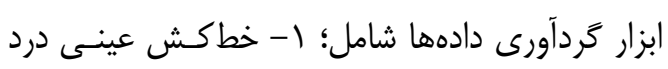

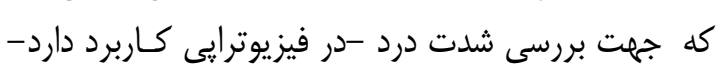

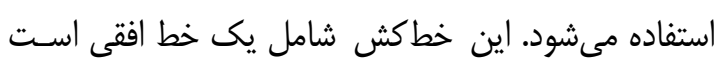

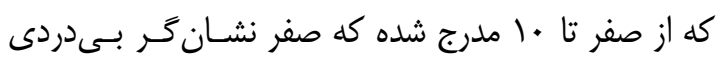

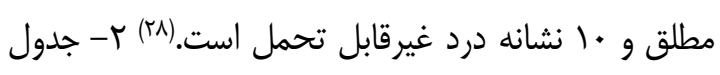

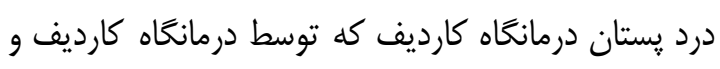
جهت تعيين مدت درد براساس تعريف ماسـتالزى دورهاى

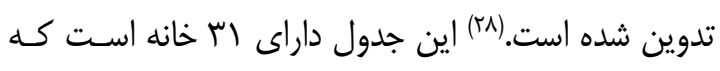

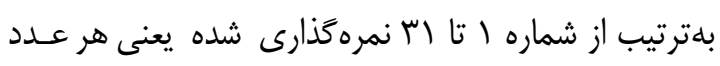
معرف همان روز از ماه است.

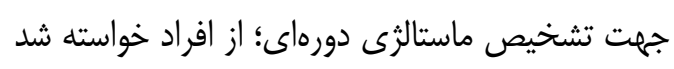

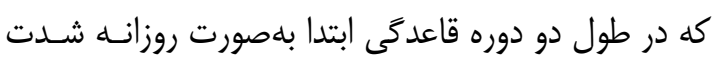

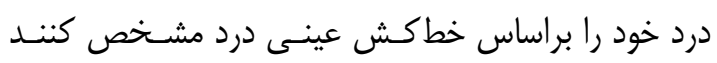




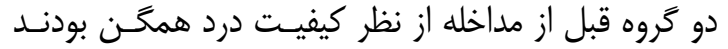

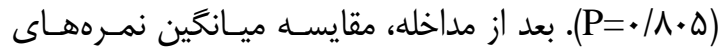

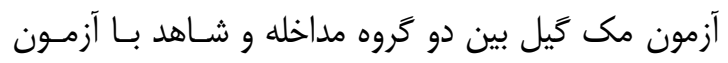

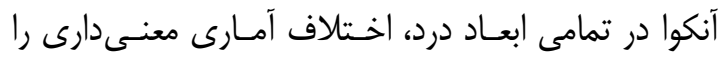
نشان داد (جدول شماره r).
نوك يستان و سابقه ضربه به پســتان تفـاوت معنسىدارى نداشتند (جدول شماره ()).

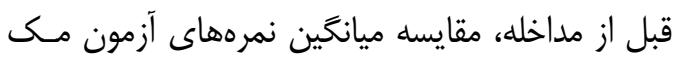

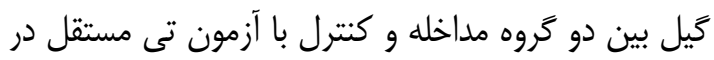
تمامى ابعاد درد اختاف آمارى معنى دارى نشان نداد يعنى

جدول (- ويزَّى هاى فردى و اجتماعى در دو َّروه مداخله و شاهد (هر تَروه +ع نفر)

\begin{tabular}{|c|c|c|c|c|c|c|c|c|}
\hline سطح معنىدارى & \multicolumn{2}{|c|}{ 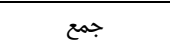 } & \multicolumn{2}{|c|}{ شاهد } & \multicolumn{2}{|c|}{ مداخله } & \multirow{2}{*}{\multicolumn{2}{|c|}{ 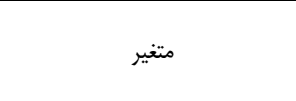 }} \\
\hline & درصد & تعداد & ل مرصد & تعداد & درصد & تعداد & & \\
\hline \multirow{2}{*}{$\cdot 189$} & $91 / 4$ & $V^{m}$ & $9 \% / 0$ & rV & 9. & ع & متأهل & \multirow{2}{*}{ تأهل } \\
\hline & $M$ & $\checkmark$ & $V / \Delta$ & $r$ & 1. & r & مجرد & \\
\hline \multirow{5}{*}{$\cdot / \& \Delta$} & 1. & $\wedge$ & $V / \Delta$ & r & $1 Y / \Delta$ & $\Delta$ & بعسواد & \multirow{5}{*}{ نحصيلات } \\
\hline & $I V / D$ & If & $1 T / \Delta$ & $\Delta$ & $r r / D$ & 9 & سيكل & \\
\hline & Fa & ع & $\Delta T / \Delta$ & r) & $r V / \Delta$ & 10 & دييله & \\
\hline & TE & $M$ & TA & 1. & $T V / D$ & 11 & 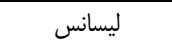 & \\
\hline & $1 / \Gamma$ & 1 & $r / \Delta$ & 1 & . & . & تحصيلات تكميلى & \\
\hline \multirow{3}{*}{$\cdot / M$} & $S V / D$ & $\Delta f$ & 90 & te & v. & rᄉ & خانهدار & \multirow{3}{*}{ 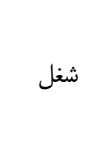 } \\
\hline & $11 / r$ & 9 & $1 T / \Delta$ & $\Delta$ & 1. & f & 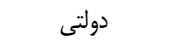 & \\
\hline & $r M / r$ & IV & $r T / Q$ & 9 & r. & $\Lambda$ & آزاد & \\
\hline \multirow{2}{*}{.109} & $\mathrm{~V} / \mathrm{\Delta}$ & gr & $\wedge$. & Tr & Va & r. & \multicolumn{2}{|c|}{ سابقه شيردهى } \\
\hline & $r T / D$ & 11 & $r$. & $\wedge$ & ro & 1. & \multicolumn{2}{|c|}{ نبود سابقه شيردهى } \\
\hline 1 & $\Delta$ & f & $\Delta$ & r & $\Delta$ & r & \multicolumn{2}{|c|}{ سابقه بيمارى خوشخيم پستان } \\
\hline 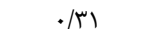 & $1 / r$ & 1 & - & . & $r / \Delta$ & 1 & \multicolumn{2}{|c|}{ سابقه ترشح از نوك يستان } \\
\hline
\end{tabular}

جدول r- مقايسه ميانكَين نمره ابعاد مختلف درد مك تَيل در تَروه مداخله و شاهد قبل و بعد از مداخله

\begin{tabular}{|c|c|c|c|c|c|c|}
\hline \multirow{3}{*}{ معنى دارى } & \multicolumn{2}{|c|}{ شاهد } & \multirow{3}{*}{ معنى دارى } & \multicolumn{2}{|c|}{ مداخله } & \multirow{3}{*}{ متغيرها } \\
\hline & بعد & قبل قبل & & بعد & قبل & \\
\hline & انحراف معيار 土ميانگين & انحراف معيار دميانخين & & انحراف معيار 土ميانكين & انحراف معيار 土ميانكَين & \\
\hline.$/ 8 \wedge \Delta$ & $\Delta / r q \pm \mid r / r$. & $\Gamma / Q \Lambda \pm \mid r / V V$ & $\cdot / \cdot Y^{*}$ & $r / r V \pm q / q \vee$ & $\Delta / \wedge) \pm 1) / १ \Delta$ & ادراك حسى درد \\
\hline.$/ 1 F \Delta$ & 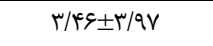 & $r / \Delta V \pm r / \cdot r$ & .1 .94 & $r / r \Delta \pm r / \kappa \Delta$ & $r / V \Psi \pm r / \Lambda V$ & ادراك عاطفى درد \\
\hline .1119 & $1 / \Delta \cdot \pm \Gamma / \cdot \cdot$ & $1 / T \Lambda \pm I / V T$ & .1 .49 & $\cdot / Q \wedge \pm 1 / r \Delta$ & $1 / r E \pm 1 / \Delta T$ & ادرك ارزيابى درد \\
\hline$\cdot \pi \cdot r$ & $r / Q T \pm r / q T$ & $r / T \Lambda \pm T / F T$ & $\cdot / \cdot \Gamma^{*}$ & $1 / \wedge r \pm r / \Delta \Delta$ & $\Gamma / \Lambda \Gamma \pm \Gamma / \Lambda \Delta$ & دردهاى متنوع و گَناكون \\
\hline אTH/. & $q / V \Lambda \pm r r / l$. & $q / \cdot I \pm r \cdot / r r$ & $\cdot / \cdot 1^{*}$ & $S / \mathcal{M} \pm 1 Q / 9 V$ & $1 . / V 9 \pm 19 / 9 V$ & نمره كل \\
\hline
\end{tabular}


مداخلههاى بىاثر خواهد شد.(") اين نتايج با مطالعه حاضر همسو است، جون در اين يزوهش با انجام مدام معاينه به زنسان

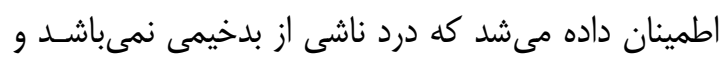

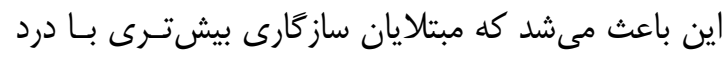

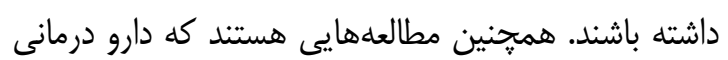

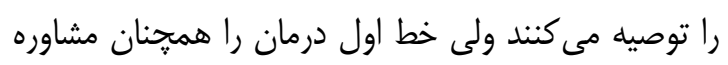

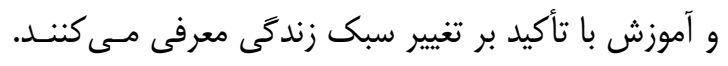

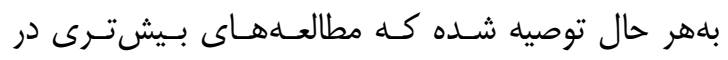
خصوص دسـتورالعمل درمـانى ماسـتالثى دورهاى انجـام شود.) ش خوص يكــى از محسـدوديتهــــا در ايـن مطالعـه، كمبــود

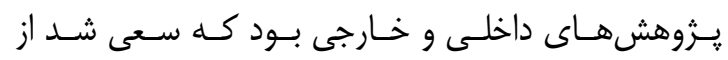

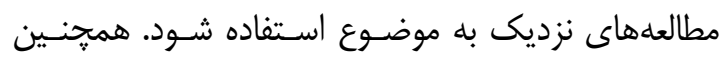

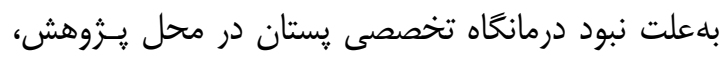

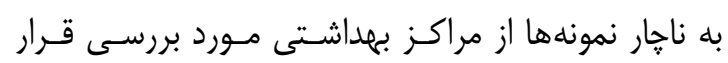

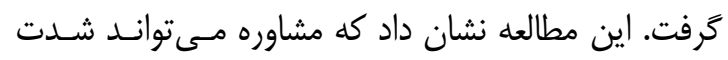

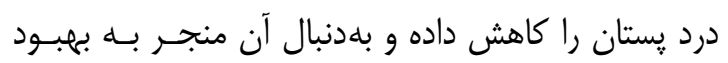

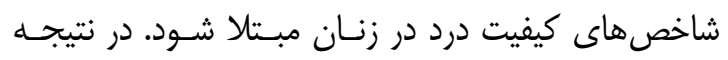

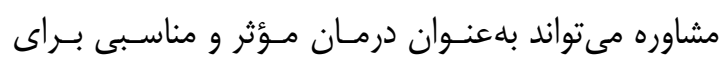

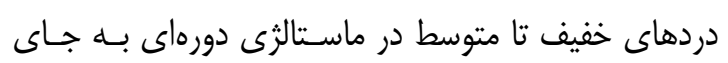

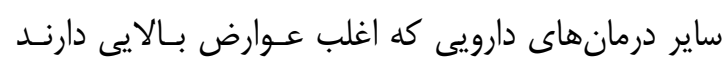
توصيه شود. مطالعههاى بيشتر در خصوص اثر مشاون داوره بر ابعاد مختلف ماستالثى دورهاى ييشنهاد مى شئود.

\section{(u)}

اين مطالعه حاصل كار يايان نامه ارشد رشته مشاوره در

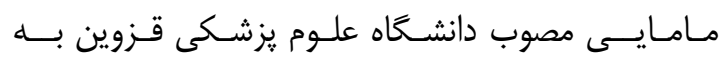

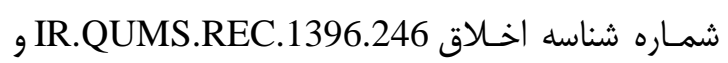

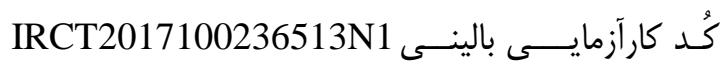

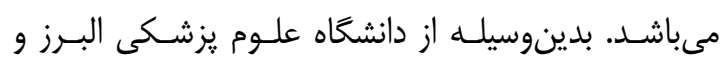

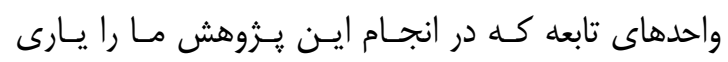
نمودند، تشكر و قدردانى مى شود.

\section{}

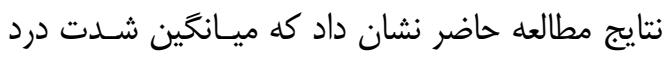

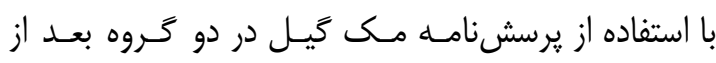

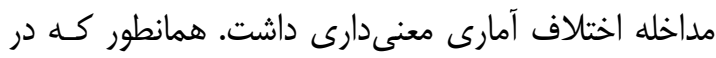

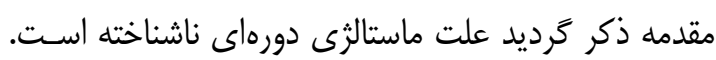

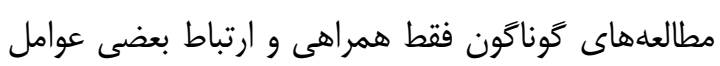

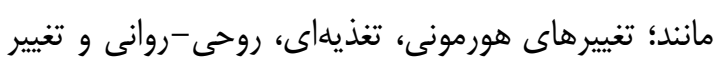

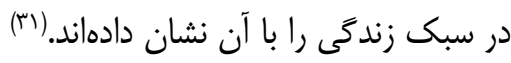

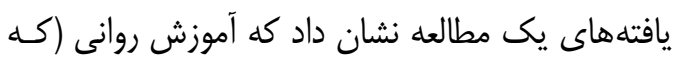

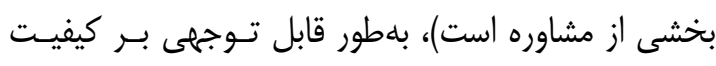

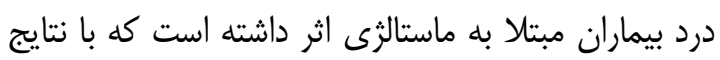

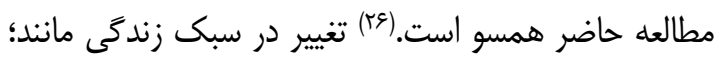

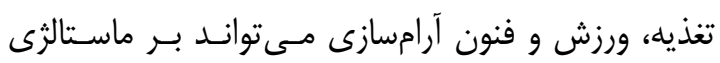

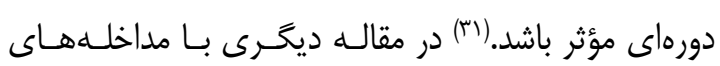
دارويى در دردهاى شديد موافق بود.

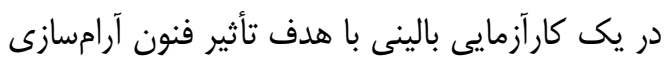

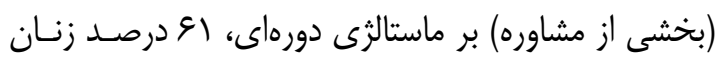

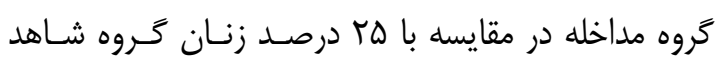

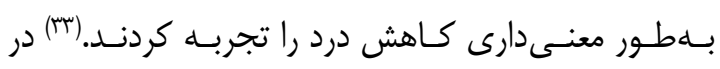

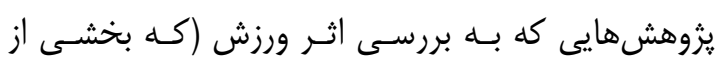

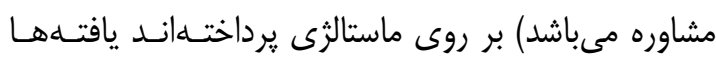

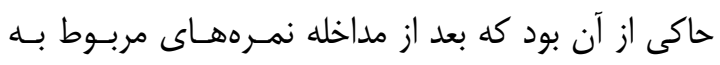

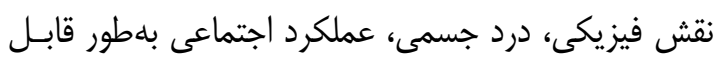

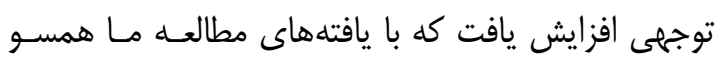

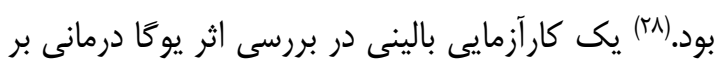

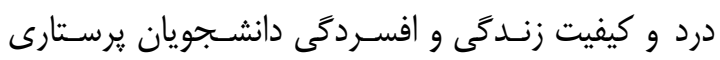

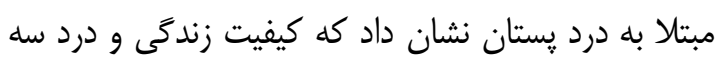

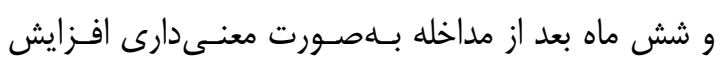

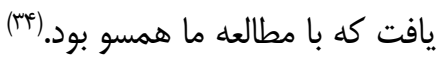

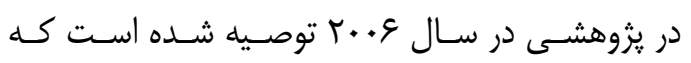

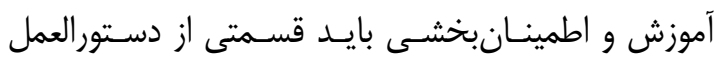

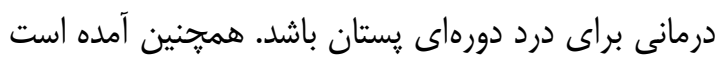

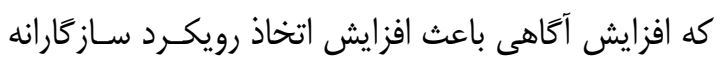

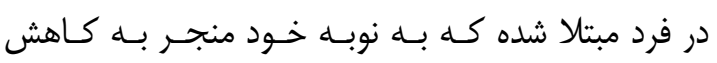


1. Parsay S, Olfati F, Nahidi S. Therapeutic effects of vitamin $\mathrm{E}$ on cyclic mastalgia. Breast J 2009; 15(5): 510-4. doi: 10.1111/j. 1524-4741.2009.00768.x.

2. Ader DN, South-Paul J, Adera T, Deuster PA. Cyclical mastalgia: prevalence and associated health and behavioral factors. J Psychosom Obstet Gynecol 2001; 22(2): 71-6. 3. Rosolowich V, Saettler E, Szuck B, Lea RH, Levesque P, Weisberg F, et al. Mastalgia. J Obstet Gynaecol Can 2006; 28(1): 49-57. doi: 10.1016/S1701-2163(16) 32027-8.

4. Milewicz A, Jedrzejuk D. Premenstrual syndrome: from etiology to treatment. Maturitas 2006; 55: S47-S54. doi: 10.1016/j. maturitas.2006.06.016.

5. Bhargav PR, Mishra A, Agarwal G, Agarwal A, Verma AK, Mishra SK. Prevalence of hypothyroidism in benign breast disorders and effect of thyroxine replacement on the clinical outcome. World J Surg 2009; 33(10): 2087-93. doi: 10.1007/ s00268-009-0143-y.

6. Sukanya R, Nagarathna R, Sandhya R, Nagendra HR. Integrated yoga therapy for mastalgia. Int J Med Sci Public Health 2016; 1(5): 162-70. doi: 10.5455/ijmsph.2016. 18122015291 .

7. Murshid KR. A review of mastalgia in patients with fibrocystic breast changes and the non-surgical treatment options. J Taibah Univ Med Sci 2011; 6(1): 1-18. doi: 10.1016/ S1658-3612(11)70151-2

8. Mirghafourvand M, Ahmadpour P, Rahi P, Salehiniya H. Relationship between depression and anxiety with the severity and length of cyclic mastalgia in women. Iran J Obstet Gynecol Infertil 2016; 18(179): 1-7. [In Persian]

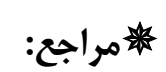

9. Colegrave S, Holcombe C, Salmon P. Psychological characteristics of women presenting with breast pain. J Psychosom Res 2001; 50(6): 303-7. doi: 10.1016/S0022-3999 (01)00196-9.

10. Morrow M. The evaluation of common breast problems. Am Fam Physician 2000; 61(8): 2371-8,2385.

11. Kaiser R, Marcus M, Blanck HM, Naughton M, Zhang RH, Henderson AK, et al. Polybrominated biphenyl exposure and benign breast disease in a cohort of US women. Ann Epidemiol 2003; 13(1): 16-23. doi: 10.1016/S1047-2797(02)00256-9.

12. Millet AV, Dirbas FM. Clinical management of breast pain: a review. Obstet Gynecol Surv 2002; 57(7): 451-61.

13. Seraji A, Salehi A, Momeni H, Kerami A, Naeimi N. The effects of evening primrose and vitex agnus on pain scale of the women with cyclic mastalgia a clinical trial. Complementary Med J 2014; 3(4): 639-53. [In Persian]

14. Shobeiri F, Oshvandi K, Nazari M. Cyclical mastalgia: prevalence and associated determinants in Hamadan city, Iran. Asian Pac J Trop Biomed 2016; 6(3): 275-8. doi: 10.1016/j.apjtb.2015.12.008.

15. Brown N, White J, Brasher A, Scurr J. The experience of breast pain (mastalgia) in female runners of the 2012 London Marathon and its effect on exercise behaviour. $\mathrm{Br} \mathrm{J}$ Sports Med 2014; 48(4): 320-5. doi: 10.1136/ bjsports-2013-092175.

16. Joshi JV, Pandey SN, Galvankar P, Gogate JA. Prevalence of premenstrual symptoms: preliminary analysis and brief review of management strategies. J Midlife Health 2010; 1(1): 30-4. doi: 10.4103/09767800.66995 . 
17. Krishnaswamy U. Profile of benign breast diseases in urban India. Indian J Surg 2003; 65(2): 178-81. doi: 10.12691/ajphr-3-4A-18.

18. Momeni H, Salihi A, Seraji A. Study of vitex agnus castus and evening primrose oil on body mass index. Complementary Med $\mathbf{J}$ 2012; 2(2): 194-203. [In Persian]

19. Scurr J, Hedger W, Morris P, Brown N. The prevalence, severity, and impact of breast pain in the general population. Breast J 2014; 20(5): 508-13. doi: 10.1111/tbj.12305.

20. Vaziri F, Samsami A, Rahimi Z, Rastgardoost N, Nick N. Prevalence, severity and factors related to mastalgia among women referring to health centers affiliated with Shiraz university of medical sciences. J Health Sci Surveillance Sys 2016; 4(2): 64-9. 21. Jaafarnejad F, Hosseini SF, Mazlom SR, Hami M. Comparison of the effect of fish oil and vitamin $\mathrm{E}$ on duration of cyclic mastalgia. Evidence Based Care J 2013; 3(1): 69-76. doi: 10.22038/EBCJ.2013.696. [In Persian] 22. Genç A, Çelebi MM, Çelik SU, Atman ED, Kocaay AF, Zergeroğlu AM, et al. The effects of exercise on mastalgia. Phys Sportsmed 2017; 45(1): 17-21. doi: 10.1080/ 00913847.2017.1252702.

23. Tofighiyan T, Najjar L, Akabery A, Shegare NM. The effect of individual counseling on the quality of life in patients with myocardial infarction. 2010; 16(54): 206-12.

24. Hosseini Birjandi M. Principles \& methods of guidance and counseling. Tehran: Roshd; 2015. 212-20.

25. Olfati F, Parsai S, Kazemnejad A, Farhad M. Comparison of two-month and four-month effect of vitamin $\mathrm{E}$ on cyclic mastalgia. J Qazvin Univ Med Sci 2006; 10(2): 60-4. [In Persian]

26. Ozenli Y, Ozturk AB, Ozturk SB, Seydaoglu G. The effects of psycho- education on the health-related quality of life on mastalgia patients. Appl Res Qual Life 2014; 9(4): 1097-105.

27. Taghizadeh Z, Shirmohammadi M, Mirmohammadali M, Arbabi M, Haghani H. The effect of counseling on premenstrual syndrome related symptoms. Hayat 2010; 15(4): 23-34. [In Persian]

28. Olfati F, Kazemi Jaliseh H, Farhad M. Survey the relationship between BMI and cyclic mastalgia. J Guilan Univ Med Sci 2009; 17(68): 50-6. [In Persian]

29. Melzack R, Katz J. McGill pain questionnaire. Encyclopedia of pain: Springer; 2013. 1792-4.

30. Khosravi M, Sadighi S, Moradi Sh, Zendehdel K. Persian-McGill pain questionnaire translation, adaptation and reliability in cancer patients: a brief report. Tehran Univ Med J 2013; 71(1): 53-8. [In Persian]

31. Smith RL, Pruthi S, Fitzpatrick LA, editors. Evaluation and management of breast pain. Mayo Clin Proc 2004; 79(3): 353-72. doi: 10.4065/79.3.353.

32. Kataria K, Dhar A, Srivastava A, Kumar S, Goyal A. A systematic review of current understanding and management of mastalgia. Indian J Surg 2014; 76(3): 217-22. doi: 10. 1007/s12262-013-0813-8.

33. Fox H, Walker L, Heys S, Ah-See A, Eremin O. Are patients with mastalgia anxious, and does relaxation therapy help? The Breast 1997; 6(3): 138-42. doi: 10.1016/ S0960-9776(97)90554-3.

34. Raghunath S, Raghuram N, Ravi S, Ram NC, Ram A. Effect of yoga therapy on quality of life and depression in premenopausal nursing students with mastalgia: a randomized controlled trial with 6-month follow-up. J Health Res Rev 2016; 3: 48-54. doi: 10.4103/2394-2010. 184229 